\title{
Tipificación y caracterización del sistema de producción del cerdo criollo de la Región Centro, México
}

\section{Typification and characterization of the creole pork production system in the Central Region, Mexico}

\begin{abstract}
Arturo Ángel-Hernández 1 (D)
Carlos Alberto García-Munguía ${ }^{1}$ (D), Alberto Margarito García-

Munguía 2 (D)

Mauricio Valencia-Posadas 1 (D)

Jesús Hernández-Ruíz (D)

Pablo Alfonso Velázquez-

Madrazo 3

${ }^{1}$ División de Ciencias de la Vida Campus Irapuato-Salamanca, Universidad de Guanajuato, km 9 carretera Irapuato Silao, Exhacienda El Copal, CP. 36824. Irapuato, Guanajuato, México.

${ }^{2}$ Centro de Ciencias Agropecuarias, Universidad Autónoma de Aguascalientes. Av Universidad 940, col. Ciudad Universitaria, CP. 20131. Aguascalientes, Aguascalientes, México.

${ }^{3}$ División de Estudios de Posgrado e Investigación. Instituto Tecnológico de Conkal. Avenida Tecnológico s/n. CP. 97345. Conkal, Yucatán, México.
\end{abstract}

*Autor de correspondencia: cagamu@hotmail.com

\section{Artículo científico}

Recibido: 15 de diciembre 2020

Aceptado: 08 de octubre 2021

Como citar: Ángel-Hernández A García-Munguía CA, García-Munguía AM, Valencia-Posadas M, HernándezRuíz J, Velázquez-Madrazo PA (2021) Tipificación y caracterización del sistema de producción del cerdo criollo de la Región Centro, México. Ecosistemas y Recursos Agropecuarios Núm. Esp. II: e2777. DOI: 10.19136/era.a8nll.2777
RESUMEN. El objetivo fue realizar la caracterización fenotípica y el sistema de producción del cerdo criollo presente en la región centro de la Sierra Madre Oriental, en los municipios de Huehuetla y San Bartolo Tutotepec (Hidalgo) y Atlequizayan, Ciudad de Cuetzalan y Zacapoaxtla (Puebla). La obtención de datos se efectuó de acuerdo con la técnica de entrevista estructurada, donde se incluyeron 55 productores dispuestos a participar y que contaran con cerdos con características de cerdo pelón mexicano y cuino. Se caracterizó la morfometría de los animales, se registró el peso corporal, altura (cruz y grupa) y largo (cuerpo, trompa y cabeza) de los individuos. Se realizó un análisis factorial por componentes principales, conglomerados y jerárquico. Para las variables no paramétricas se utilizó estadísticas descriptiva y análisis de varianza multifactorial. Se identificaron dos tipos de sistemas de producción de traspatio para la cría de Cerdo Criollo en la Región Centro de la Sierra Madre Oriental, el primero es para autoconsumo, el segundo se diferencia por la incorporación de mejoras en el manejo, en la forma de producción, enfoque expansivo. Además, los cerdos criollos de la región se encuentran diferenciados en su morfometría dependiendo de su lugar de origen.

Palabras clave: Cerdo Pelón mexicano, cuino, porcinocultura, recursos zoogenéticos, traspatio.

ABSTRACT. The objective of the present work was to make a phenotypic characterization and the production system of the Creole pig present in the central region of the Sierra Madre Oriental, in the municipalities of Huehuetla and San Bartolo Tutotepec (Hidalgo) and Atlequizayan, Ciudad de Cuetzalan and Zacapoaxtla (Puebla). Data collection was carried out according to the structured interview technique, where 55 producers willing to participate and who had pigs with cerdo pelon mexicano and cuino characteristics were included. The animals were characterized morphometrically, body weight, heights (withers and rump), lengths (body, trunk and head) were recorded. A factorial analysis by principal components, clusters and hierarchical was carried out. Descriptive statistics and multifactorial analysis of variance were used for non-parametric variables. Two types of backyard production systems were identified for the breeding of Criollo Pigs in the Central Region of the Sierra Madre Oriental, the first is for self-consumption, the second is differentiated by the incorporation of improvements in management, in the form of production, expansive approach. In addition, the native pigs of the region are differentiated in their morphometry depending on their place of origin.

Key words: Cerdo Pelón mexicano, cuino, pig farming, Animal genetic resources, backyard. 


\section{INTRODUCCIÓN}

El Cerdo Pelón Mexicano (CPM) y el Cerdo Cuino (CC) son razas naturalizadas en el país que representan poblaciones descendientes del cerdo Ibérico (Burgos-Paz et al. 2013, Ogata 2019) y de los cerdos asiáticos introducidos por los españoles durante la conquista, desde entonces forman parte del mestizaje cultural mexicano (Mireles et al. 2015). Estos cerdos se fueron distribuyendo en diversas regiones del país y en una gran variación de climas a los que se fueron adaptando y distanciando genéticamente (Lemus-Flores et al. 2020). En la actualidad se tienen descritos en las zonas tropicales de la región del Golfo y el Pacifico (Fuentes-Mascorro et al. 2011), las características fenotípicas que distinguen al CPM es escases de pelo en la mayoría del cuerpo y un color gris a negro (Lemus et al. 2003, ÁngelHernández et al. 2020), el CC son peludos y robustos, además poseen rusticidad y adaptabilidad al medio en donde se desarrollan. La producción de los cerdos se da en forma tradicional principalmente en el traspatio y es parte de la cultura de algunas comunidades del Sur-Sureste del país donde se conserva inmerso en las tradiciones y gastronomía local (Sierra et al. 2016). Una problemática actual que se presenta en las poblaciones que aún se encuentran, es la disminución de la variabilidad genética, causada por los fenómenos de cruzamientos y/o sustitución con razas comerciales (Clemente et al. 2008).

Los trabajos dirigidos al estudio de los sistemas de producción de CPM y CC se han enfocado en áreas geográficas muy específicas como en la Península de Yucatán y los estados de $\mathrm{Na}$ yarit, Oaxaca, Chiapas y Veracruz (Lemus et al. 2003, Fuentes-Mascorro et al. 2011). Mientras que del resto del país se tiene poca información o se desconoce la situación actual, como ocurre en el Centro del país donde existen poblaciones de estos animales, pero no se han caracterizado.

La FAO (2007) refiere que para el uso adecuado de los recursos zoogenéticos, éstos deben ser caracterizados y conservados a fin de tenerlos accesibles para su aprovechamiento. Por lo antes mencionado es relevante contar con estudios que describan el fenotipo de estos animales y los sistemas de producción donde se desarrollan. El objetivo del presente trabajo fue hacer una tipificación y caracterización del sistema de producción del cerdo criollo de la región centro de la Sierra Madre Oriental.

\section{MATERIALES Y MÉTODOS}

\section{Localización del área de estudio}

El trabajo se realizó en los municipios de Huehuetla y San Bartolo Tutotepec en el estado de Hidalgo y Atlequizayan, Ciudad de Cuetzalan y Zacapoaxtla en el estado de Puebla, estas comunidades pertenecen geográficamente a una franja homogénea de la Sierra Madre Oriental que se caracteriza por un clima semicálido húmedo, con una temperatura media anual que oscila entre 17 a $23^{\circ} \mathrm{C}$, con una precipitación de 1000 a 3000 milímetros cúbicos por año y altura de 400 hasta los $1600 \mathrm{msnm}$ (Suárez-Mota et al. 2017). El estudio se realizó en cinco visitas a la región durante el periodo de 2018 al 2020.

\section{Diseño de estudio y muestreo}

Los datos se obtuvieron de acuerdo con la técnica de entrevista estructurada propuesta por Blanchet et al. (1989). Se incluyeron 55 productores dispuestos a participar y que contaran con cerdos que cumplieran con las características fenotípicas del CPM y CC (Ausencia de pelo y color de piel gris oscuro, o abundante pelo y robusto) (Lemus et al. 2003, Ángel-Hernández et al. 2020). Se aplicó un cuestionario de 96 reactivos comprendiendo los apartados de: Características sociodemográficas del productor, composición de la piara, manejo general, alimentación, sanidad, reproducción, genética, comercialización y características fenotípicas. Para estas últimas características se tomaron en cuenta 121 hembras y 36 machos, registró el peso corporal con una báscula con capacidad de $200 \mathrm{~kg}$, altura a la cruz, altura a la grupa, largo del cuerpo, largo de trompa y largo de cabeza $(\mathrm{cm})$, las cuales fueron medidas con cinta métrica, según las guías de la FAO (FAO 2011, FAO 2012). 


\section{Análisis estadísticos}

La estratificación de los productores se realizó con un análisis multivariado utilizando un análisis factorial por componentes principales, conglomerados jerárquicos y de K-medias, en tres pasos. El análisis factorial por componentes principales, se utilizó para reducir el número de variables (51) cuantitativas (Tabla 1), con los valores originales (Gelasaki et al. 2012), mediante la construcción de factores que expliquen la mayor varianza en el análisis global (Castaldo et al. 2006). El análisis de conglomerados jerárquico se utilizó para identificar el número de grupos de productores de forma gráfica, basado en el algoritmo de Ward (García y Calle 2013) para encontrar el punto de corte en el dendrograma (Figura 1). Las variables utilizadas fueron los factores obtenidos en el análisis factorial por componentes principales, y se estandarizaron con la media y desviación estándar. Los análisis estadísticos se realizaron con el programa estadístico SAS.

\section{RESULTADOS}

En la región centro de la sierra madre oriental de los estados de Hidalgo y Puebla, los sistemas de CPM son en su totalidad de traspatio, con edad promedio de los productores de 43 años, en su mayoría hombres $(74.5 \%)$ que no superan los dos años de experiencia en el cuidado de estos animales. Las personas que crían a los cerdos criollos cuentan con estudios universitarios (56.3\%), secundaria $(20 \%)$ y primaria $(23.6 \%)$, no se encontró a personas analfabetas. Esta actividad es una fuente secundaria de ingresos (96.3\%), la mayoría se dedican a la agricultura $(67.2 \%)$, son obreros $(20 \%)$ o tienen otros oficios (12.8\%). El $60 \%$ de las personas iniciaron con la crianza de cerdos para obtener ingresos adicionales y el $40 \%$ refiere tener especial interés y gusto por esta raza. En general la principal problemática para iniciar con el sistema de producción fue la dificultad de encontrar la raza $(60 \%)$ y en segundo lugar el nivel de consanguinidad $(40 \%)$, debido a los pocos criadores y ejemplares que se encuentran en la zona.

En la región se encontraron dos razas criollas, el CPM (89\%) y en menor proporción el CC (5.4\%).
Con respecto a las características del sistema de producción, estos están conformados por más de dos hembras productoras con un verraco, en algunos casos se cuenta con una hembra y un macho de reemplazo, en promedio se tienen cinco animales, el número de crías es de cuatro a cinco lechones (Tabla 1). Los animales se encuentran permanente en los corrales y tienen como finalidad el autoconsumo y el pie de cría $(76.3 \%)$. Un porcentaje menor se destina a la venta de lechones destetados (23.6\%), en general no se llevan registros productivos ni se tiene un método de identificación convencional. Cuando los criadores adquieren animales, lo hacen en comunidades aledañas $(87.2 \%)$ o tienen que ir fuera del estado (12.7\%).

El manejo en los lechones es la castración a machos antes del destete (1.8 meses), el destete a los dos meses de edad y no descolmillan. Otro parámetro que se consideró fue el uso de estiércol, en su mayoría lo usan como fertilizante en los cultivos $(53.7 \%)$, el resto refiere no utilizarlo $(46.2 \%)$. La alimentación de los cerdos es a base de maíz, desechos agrícolas y de cocina (67.2\%), en algunos casos el maíz se mezcla con alimento comercial (16.3\%) o salvado (12.7\%). El manejo sanitario, es mínimo, la mayoría no desparasitan (81.8\%) y no vacunan.

Los productores realizan la selección de los animales (97.2\%), la cual se basa en el gusto de cada persona, para la selección de sementales principalmente se considera la actitud del animal. La vida productiva de los reproductores es de siete años o de siete partos para las hembras. Los sementales identificados en los sistemas productivos tienen un año de trabajo y más de cinco años de edad (89\%). La edad al primer parto es de seis a ocho meses, no se registró la presencia de abortos, no se dio asistencia en el parto y se contabilizaron de seis a nueve lechones por parto con dos partos por año, con un lechón muerto al destete en promedio. La comercialización de los cerdos se realiza por lo menos una vez al año, se venden de uno a 10 animales (75\%) y en menor proporción se comercializan de 11 a 50 cerdos (25\%), los ingresos por la venta se destinan principalmente para cubrir los gastos que genera la crianza de los cerdos. Los produc- 
Tabla 1. Variables cuantitativas por componentes utilizadas para la caracterización y tipología de los sistemas de producción del Cerdo Criollo Mexicano en la Región Centro de la Sierra Madre Oriental, México.

\begin{tabular}{llcc}
\hline Componentes & Variables & Media & $\mathrm{DE}$ \\
\hline Datos del productor & Edad & 43.65 & 10.27 \\
& Años como criador & 1.82 & 0.64 \\
Características del sistema & Número de lechones & 4.93 & 0.60 \\
& Número de verracos & 1.20 & 0.62 \\
& Número de hembras & 2.29 & 1.05 \\
& Número de machos de reemplazo & 0.43 & 0.24 \\
& Número de hembras de reemplazo & 0.66 & 0.56 \\
Manejo general & Número total & 4.29 & 1.27 \\
& Edad al destete (meses) & 2.00 & 0.00 \\
& Edad de la castración (meses) & 1.85 & 0.36 \\
\hline
\end{tabular}

DE: Desviación estándar, $\mathrm{n}=55$.

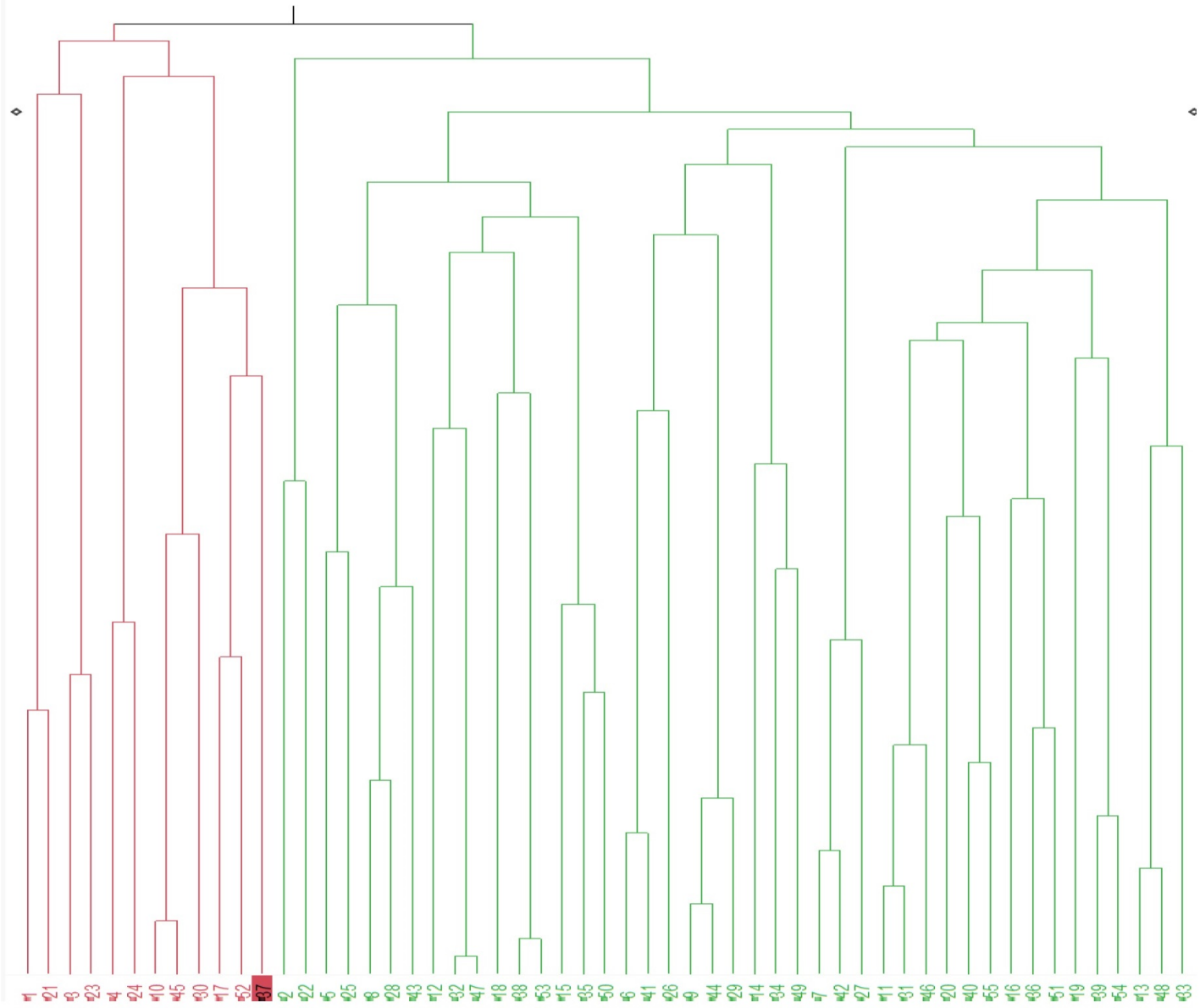

Figura 1. Dendrograma de las tipologías de los sistemas de producción de Cerdo Criollo Mexicano de la Región Centro de la Sierra Madre Oriental, México.

tores manifestaron la falta de un mercado constante, la venta solo es por temporadas (Tabla 2).

De las 51 variables evaluadas se seleccionaron
16 (Tabla 3), de mayor importancia con los eigenvalores diferentes a cero $(0.22$ a 0.49$)$. A partir del análisis factorial con las variables seleccionadas 
Tabla 2. Variables cualitativas por componentes utilizadas para la caracterización y tipología de los sistemas de producción del Cerdo Criollo Mexicano en la Región Centro de la Sierra Madre Oriental, México.

\begin{tabular}{|c|c|c|c|}
\hline Componentes & Variables & Clases & $\%$ \\
\hline \multirow[t]{15}{*}{ Datos del productor } & \multirow{2}{*}{ Genero } & Masculino & 74.5 \\
\hline & & Femenino & 25.4 \\
\hline & \multirow[t]{3}{*}{ Escolaridad } & Primaria & 23.6 \\
\hline & & Secundaria & 20 \\
\hline & & Licenciatura & 56.3 \\
\hline & \multirow{2}{*}{$\begin{array}{l}\text { Fuente de ingresos que } \\
\text { representan los cerdos }\end{array}$} & Primaria & 3.6 \\
\hline & & Secundaria & 96.3 \\
\hline & \multirow[t]{4}{*}{ Realiza otra actividad } & Agrícola & 67.2 \\
\hline & & Comerciante & 3.6 \\
\hline & & Obrero & 20 \\
\hline & & Otra & 9.1 \\
\hline & \multirow[t]{2}{*}{ Motivo para iniciar } & Economía & 60 \\
\hline & & Gusto & 40 \\
\hline & \multirow[t]{2}{*}{ Problemas para iniciar } & Conseguir los animales & 60 \\
\hline & & Consanguinidad & 40 \\
\hline \multirow[t]{10}{*}{ Características del sistema } & Como tiene a los animales & En corrales & 100 \\
\hline & \multirow[t]{3}{*}{ Tipo de cerdo } & Cuino & 5.4 \\
\hline & & Cerdo pelón mexicano & 89.1 \\
\hline & & Cuino y Cerdo pelón mexicano & 5.4 \\
\hline & \multirow[t]{2}{*}{ Fin de la explotación } & Autoconsumo/Ahorro & 23.6 \\
\hline & & Venta & 76.3 \\
\hline & \multirow[t]{2}{*}{ Adquisición } & En comunidades aledañas & 87.2 \\
\hline & & Fuera del estado & 12.7 \\
\hline & Tipo de registros & No llevan registro & 100 \\
\hline & Identificación de cerdos & No identifican & 100 \\
\hline \multirow{5}{*}{ Manejo general } & Descolmilla & No & 100 \\
\hline & Castración & $\mathrm{Si}$ & 100 \\
\hline & \multirow[t]{2}{*}{ Uso del estiércol } & Fertilizante & 46.2 \\
\hline & & No lo usan & 53.7 \\
\hline & Desteta & $\mathrm{Si}$ & 100 \\
\hline \multirow[t]{6}{*}{ Alimentación } & \multirow[t]{4}{*}{ Con que alimenta } & Maíz & 3.6 \\
\hline & & Maíz y alimento comercial & 16.3 \\
\hline & & Maíz y salvado & 12.7 \\
\hline & & Maíz y desperdicio de cosecha y cocina & 67.2 \\
\hline & Suplementa hembras lactantes & No & 100 \\
\hline & Proporciona forrajes & $\mathrm{Si}$ & 100 \\
\hline Sanidad & Desparasita & $\mathrm{Si}$ & 18.2 \\
\hline & & No & 81.8 \\
\hline & Vacuna & No & 100 \\
\hline & Frecuencia de vacunación & Ninguna & 100 \\
\hline & Muerte de cerdos & $\mathrm{Si}$ & 96.4 \\
\hline & & No & 3.6 \\
\hline Reproducción & Realiza selección en su piara & $\mathrm{Si}$ & 92.6 \\
\hline & & No & 7.3 \\
\hline & Apoya en el parto & No & 100 \\
\hline & Selecciona el semental & Por la actitud & 100 \\
\hline & Edad al primer parto & 6 a 8 meses & 96.4 \\
\hline & & más de 8 meses & 3.6 \\
\hline & Lechones por parto & 6 a 9 lechones & 100 \\
\hline & Partos por año & 2 veces & 100 \\
\hline & Lechones muertos al destete & 1 lechón & 100 \\
\hline & Edad del semental & 3-5 años & 10.9 \\
\hline & & más de 5 años & 89.1 \\
\hline & Tiempo de uso del semental & 1 año & 10 \\
\hline & & 2 años & 90 \\
\hline & Tiempo para desechar al semental & 5-7 años & 100 \\
\hline & Tiempo para desechar a las hembras más de & 7 partos & 100 \\
\hline & Abortos & No & 100 \\
\hline Comercialización & Vende los cerdos & $\mathrm{Si}$ & 100 \\
\hline & Problemas para vender & No hay venta contante & 100 \\
\hline & Cada cuando vende & 1 vez al año & 100 \\
\hline & Motivo de la venta & para cubrir gastos de los cerdos & 100 \\
\hline & Cuanto vende al año & $1-10$ animales & 75 \\
\hline & & $11-50$ animales & 25 \\
\hline
\end{tabular}

se extrajeron tres factores que presentaron valores propios mayores a 1 basados en el criterio de raíz latente, lo que explican el $96.9 \%$ de la variación total de las variables originales.

En el dendograma se diferencian dos sistemas de producción de traspatio para el cerdo criollo en la región (Figura 1), los cuales son el sistema de pro- ducción de traspatio de autoconsumo (SPTA) y el sistema de producción de traspatio expansivo (SPTE). Además, se describen las variables que diferencian los sistemas de producción como resultado del análisis factorial por componentes principales. En el SPTA predominan las mujeres como productores (25.4\%), con escolaridad de primaria (23.6\%) y secundaria 
Tabla 3. Cargas factoriales de las variables que componen los factores definidos para los sistemas de producción del Cerdo Criollo Mexicano en la Región Centro de la Sierra Madre Oriental, México.

\begin{tabular}{|c|c|c|c|}
\hline \multirow[b]{2}{*}{ Variables* } & \multicolumn{3}{|c|}{$\begin{array}{l}\text { Componentes principales } \\
\qquad\left({ }^{\star \star} \text { Eigenvalores }\right)\end{array}$} \\
\hline & 1 & 2 & 3 \\
\hline Género & -0.0603 & -0.4055 & 0.0967 \\
\hline Escolaridad & 0.0185 & 0.3778 & -0.2401 \\
\hline Edad & 0.2364 & 0.2278 & 0.0262 \\
\hline Número de hembras & 0.2063 & 0.1433 & -0.2553 \\
\hline Número de verracos & 0.0543 & 0.1765 & 0.4808 \\
\hline Número de machos de reemplazo & 0.0200 & 0.2342 & -0.4467 \\
\hline Número de hembras de reemplazo & -0.0087 & 0.1635 & 0.4955 \\
\hline Número de Lechones & 0.1664 & -0.2894 & 0.0095 \\
\hline Número total & 0.1805 & 0.3052 & 0.0924 \\
\hline Tipo de cerdo & -0.2164 & 0.2249 & 0.2018 \\
\hline Adquisición & 0.2803 & 0.0605 & 0.0270 \\
\hline Fuente de ingresos & -0.2822 & 0.0046 & -0.0499 \\
\hline Realiza otra actividad & 0.2585 & -0.1315 & -0.1527 \\
\hline Años como criador & 0.2753 & -0.1039 & -0.0405 \\
\hline Fin de la explotación & -0.1895 & -0.1731 & -0.2606 \\
\hline Con que alimenta & 0.2822 & -0.0046 & 0.0499 \\
\hline Varianza explicada (\%) & 56.01 & 25.39 & 15.53 \\
\hline
\end{tabular}

(20\%), se dedican a la actividad agrícola (67.2\%) y al comercio como actividad secundaria (3.6\%). El $40 \%$ iniciaron con la crianza de cerdos por gusto y tienen como fin de la explotación producir lechones al destete $(23.6 \%)$, la adquisición de nuevos animales se hace en comunidades aledañas (12.7\%). En cuanto al estiércol no se le da ningún tipo de uso, y se alimentan de maíz y desperdicio de cosecha y cocina, no desparasitan y las ventas anuales van de 1 a 10 animales (75\%). En contraste, en el SPTE los productores son hombres $(74.5 \%)$ con estudios de licenciatura (56.3\%), se dedican a la actividad agrícola, pecuaria y obrera (20\%). El motivo de iniciar con la crianza de cerdos fue económica con el objetivo de producción para la venta $(76.3 \%)$, los animales los adquieren fuera del estado. En cuanto al uso del estiércol lo utilizan como fertilizante obteniendo así un subproducto, los animales son alimentados con maíz (3.6\%), maíz y alimento comercial (16.3) y maíz y salvado (12.7), se usó desparasitante (18.2\%) y venden de 11 a 50 cerdos al año (25\%).

Los datos morfométricos de los cerdos presentan diferencias según el lugar de origen, los cerdos del estado de Hidalgo son más pesados que los del estado de Puebla, con lo que respecta a la longitud también se presentan diferencias, la longitud de cabeza y hocico son más largas en los animales de Puebla, pero el cuerpo es corto en comparación con los de Hidalgo, para la variable de altura a la cruz no se presentan diferencias, pero si las hay en altura a la grupa al igual que el perímetro del pecho. Los animales de ambos estados presentan dimorfismo sexual favoreciendo a los machos (Tabla 4).

\section{DISCUSIÓN}

Los datos muestran que en el SPTE los hombres son quienes se dedican a la crianza de cerdos y estos cuentan con estudios universitarios, lo que indica que esta es una actividad con un gran interés y perspectiva económica en el CPM. ya que la consideran una fuente real de ingreso sobrepasando el concepto de autoconsumo debido a la tendencia del mercado donde se busca bienestar animal y productos de calidad (Ángel-Hernández et al. 2020). Esta tendencia puede deberse a la perspectiva negativa que se ha formado por parte 
Tabla 4. Características morfométricas del Cerdo Criollo Mexicano en relación con su origen y sexo.

\begin{tabular}{|c|c|c|c|c|c|c|c|c|}
\hline & Hidalgo & Puebla & Machos & Hembras & Hidalgo/Hembras & Hidalgo/Machos & Puebla/Hembras & Puebla/Machos \\
\hline & Media $\pm \mathrm{EE}$ & Media $\pm \mathrm{EE}$ & Media $\pm \mathrm{EE}$ & Media $\pm \mathrm{EE}$ & Media $\pm \mathrm{EE}$ & Media $\pm \mathrm{EE}$ & Media $\pm \mathrm{EE}$ & Media $\pm \mathrm{EE}$ \\
\hline $\begin{array}{l}\text { Peso kg } \\
\text { Longitud }\end{array}$ & $35.69 \pm 0.21^{* *}$ & $31.85 \pm 0.18^{* *}$ & $36.57 \pm 0.22^{* *}$ & $30.97 \pm 0.17^{* *}$ & $32.79 \pm 0.25^{N S}$ & $38.57 \pm 0.34^{N S}$ & $29.15 \pm 0.22^{N S}$ & $34.55 \pm 0.27^{N S}$ \\
\hline Cabeza & $24.66 \pm 0.09^{* *}$ & $27.93 \pm 0.07^{* *}$ & $27.52 \pm 0.09^{* *}$ & $25.06 \pm 0.07^{* *}$ & $23.47 \pm 0.11^{N S}$ & $25.84 \pm 0.14^{N S}$ & $26.66 \pm 0.09^{N S}$ & $29.2 \pm 0.12^{N S}$ \\
\hline Hocico & $11.04 \pm 0.14^{* *}$ & $12.6 \pm 0.12^{* *}$ & $12.4 \pm 0.15^{* *}$ & $11.24 \pm 0.11^{* *}$ & $10.67 \pm 0.17^{\star}$ & $11.42 \pm 0.23^{\star}$ & $11.82 \pm 0.15^{\star}$ & $13.37 \pm 0.18^{\star}$ \\
\hline $\begin{array}{l}\text { Cuerpo } \\
\text { Altura }\end{array}$ & $95.42 \pm 0.2^{* *}$ & $80.03 \pm 0.16^{* *}$ & $89.64 \pm 0.2^{* *}$ & $85.81 \pm 0.16^{* *}$ & $93.58 \pm 0.24^{N S}$ & $97.26 \pm 0.32^{N S}$ & $78.04 \pm 0.21^{N S}$ & $82.03 \pm 0.26^{N S}$ \\
\hline Cruz & $56.75 \pm 0.1^{N S}$ & $56.92 \pm 0.08^{N S}$ & $58.13 \pm 0.10^{* *}$ & $55.53 \pm 0.08^{* *}$ & $55.5 \pm 0.12^{N S}$ & $58 \pm 0.16^{N S}$ & $55.57 \pm 0.1^{N S}$ & $58.27 \pm 0.13^{N S}$ \\
\hline Grupa & $22.22 \pm 0.1^{* *}$ & $17.74 \pm 0.08^{* *}$ & $21.23 \pm 0.1^{* *}$ & $18.72 \pm 0.08^{* *}$ & $20.97 \pm 0.12^{N S}$ & $23.47 \pm 0.16^{N S}$ & $16.48 \pm 0.1^{N S}$ & $19 \pm 0.13^{N S}$ \\
\hline
\end{tabular}

del consumidor en torno a la porcinocultura intensiva donde se generaliza el uso de productos químicos sintéticos (Cancho et al. 2000) y las condiciones de estrés a las que son sometidos los animales, lo que se contrapone a los intereses de los consumidores que cada vez centran más su atención en la forma de producir la carne (FAO 2014). Debido a esto la preferencia por productos y subproducto porcinos asociados a estilos de vida más saludables y procesos de calidad justifican un valor agregado para estos productos (Savón 2014, Contino-Esquijerosa et al. 2017), donde la definición de calidad y seguridad se fundamenta en el conocimiento de los procesos nutritivos e higiénico-toxicológicos en los que se basa su producción (Castro y Rodríguez 2005).

El grupo SPTA presentó características de un sistema de traspatio donde el cerdo CPM se utiliza para autoconsumo, en este sistema los productores son personas con escolaridad mínima (primaria y secundaria), lo anterior coincide con lo que se ha observado en sistemas de traspatio del país; principalmente en los estados de Yucatán y Nayarit (Lemus y Ly 2010, Sierra et al. 2016). En los estados de Oaxaca (Fuentes-Mascorro et al. 2011) y Chiapas (Perezgrovas et al. 2007) también se ha reportado la presencia de sistemas de producción para autoconsumo, a diferencia de esta investigación estos se encuentran en zonas indígenas y las mujeres son las encargadas del sistema productivo. En el ámbito internacional se han realizado estudios enfocados en cerdos autóctonos, en Portugal se reportó un sistema tradicional y uno semi-intensivo donde los productores son hombres de aproximadamente 47 años con una tendencia enfocada a la comercialización y no al autoconsumo (Paixão et al. 2018). En esta ten- dencia comercial se están dirigiendo los sistemas de producción en México como se describe en este estudio donde tenemos un sistema tradicional enfocado al autoconsumo y otro encaminado a la obtención de mejores parámetros en la producción. Además, es de resaltar que en Europa la crianza de cerdos criollos es una opción para una producción de carne de mejor calidad, respetando el bienestar animal (Petrovic et al. 2013, Maroto-Molina et al. 2018).

Estudios realizados en unidades de producción rural o de traspatio en países como Colombia (Díaz et al. 2011), Nicaragua (Hernández-Baca et al. 2017), China (Riedel et al. 2014), Indonesia (Leslie et al. 2015), Perú (Morales et al. 2014), Uruguay (Blumetto et al. 2013) y Australia (Schembri et al. 2015), coinciden con el grupo SPTA donde los cerdos criollos se utilizan como una opción de autoconsumo y ahorro. Las poblaciones de cerdos criollos fenotípicamente son muy heterogéneos, la variabilidad morfológica puede atribuirse a la diferencia en los sistemas de manejo, ambientes en donde se desarrollan las subpoblaciones y a la variabilidad genética (Pérez et al. 2015, Martínez et al. 2016). Por lo que las medidas zoometrías son importantes para determinar los estándares de la forma del cuerpo animal (Linares et al. 2011), ya que estas características están influenciadas por el desarrollo de los huesos y por la deposición de musculo y grasa (Salamanca-Carreño et al. 2020). Estudios realizados en poblaciones de cerdos criollos de 18 estados del país determinaron para cerdo pelón mexicano tiene un peso promedio de $48.06 \pm 6.17 \mathrm{~kg}$ (Martínez et al. 2016), mientras que las medias obtenidas en los pesos de cerdos criollos de Guatemala fue de $45.52 \mathrm{~kg}$ en machos y $45.46 \mathrm{~kg}$ para hembras (Lorenzo et al. 2012). Por 
lo que estos resultados son mayores a los obtenidos en este estudio, diferencias que pueden deberse a la edad de los animales, alimentación, genética, ambientales, entre otras. Para la variable longitud de cuerpo los animales más largos se tuvieron en los sistemas de producción del estado de hidalgo con valores de $97.26 \pm 0.32 \mathrm{~cm}$. Al respecto Pujada et al. (2018) reportan que en Cerdo Criollo Alto Andino la longitud del cuerpo media fue de $71.0 \pm 1.0$ para hembras y $72.0 \pm 1.0 \mathrm{~cm}$ para machos. Al respecto la mayor longitud de cuerpo encontrada en el presente trabajo indica una mejor constitución y adaptación al medio ambiente (Vargas et al. 2015). Mientras que Ramírez-Reyes et al. (2020) reportan diferencias entre la longitud de cabeza de machos y hembras con valores de $28.26 \pm 0.84 \mathrm{~cm}$ y $31.17 \pm 0.82 \mathrm{~cm}$, respectivamente, valores que son similares a lo reportado por Martínez et al. (2016) con $27.22 \pm 1.4$ $\mathrm{cm}$, con diferencias en el sexo siendo, mayores los machos (36.57 $\pm 0.22 \mathrm{~kg}$ ) que las hembras (30.97 \pm $0.17 \mathrm{~kg}$ ) y por lugar de origen los cerdos de Hidalgo presentan mayores valores $(35.69 \pm 0.21 \mathrm{~kg})$, que los de Puebla (31.85 $\pm 0.18 \mathrm{~kg})$.

La media de alzada de cruz en el cerdo alto Andino es de $45.6 \pm 1.9 \mathrm{~cm}$ para hembras y $46.9 \pm$ $2.1 \mathrm{~cm}$ para machos (Pujada et al. 2018), característica que esta poco influenciada por las condiciones del medio. Por lo que constituye un carácter morfológico estable y adecuado para delimitar diferencias o similitudes entre cerdos provenientes de diferentes orígenes (Lorenzo et al. 2012). Los cerdos con mayor circunferencia de pecho $103.63 \pm 0.79 \mathrm{~cm}$ fueron los del estado de Hidalgo lo que indica que son los animales más largos. Al respecto, Ramírez-Reyes et al. (2020) reportan para cerdo pelón mexicano de la Península de Yucatán valores de circunferencia de pecho de $82.91 \pm 3.02$ en machos y de $89.79 \pm 3.03$ en hembras, lo que indica que el cerdo criollo presente en el centro del país es de mayor tamaño a los reportados en el Sureste Mexicano. Mientras que Revidatti (2009) refiere que diámetros dorsoesternales cortos y extremidades de mayor longitud son características que se presentan en animales rústicos o pocos mejorados y que cuya aptitud es la obtención de carne magra.

\section{CONCLUSIONES}

En la región centro de la Sierra Madre Oriental, Se tipificó un sistema de producción de traspatio, con dos variantes, la primera un sistema de producción de traspatio de autoconsumo (SPTA) y la segunda un sistema de producción de traspatio expansivo (SPTE). Se diferencian por el género, escolaridad, edad, actividad económica, actividades secundarias del productor, así como por fin de la producción, lugar de adquisición, alimentación y manejo de excretas principalmente. Además, los cerdos criollos se encuentran diferenciados en su morfometría dependiendo del lugar de origen.

\section{LITERATURA CITADA}

Ángel-Hernández A, García MCA, García MAM, Ortíz OJR, Sierra VAC, Morales-Flores S (2020) Sistema de producción del Cerdo Pelón Mexicano en la Península de Yucatán. Nova Scientia 24: 1-21. DOI: 10.21640/ns. v12i24.2234.

Blanchet A, Ghiglione R, Massonat J, Trognon A (1989) Técnicas de investigación en ciencias sociales. Narcea ediciones. Madrid, España. 60p.

Blumetto V, Calvet SS, Estellés BF, Villagrá GA (2013) Comparison of extensive and intensive pig production systems in Uruguay in terms of ethologic, physiologic and meat quality parameters. Revista Brasileira de Zootecnia 42: 521-529

Burgos-Paz W, Souza CA, Megens HJ, Ramayo-Caldas Y, Melo M, Lemus-Flores C, Caal E, Soto HW, Martínez R, Álvarez LA (2013) Porcine colonization of the Americas: a 60k SNP story. Heredity 110: 321-330. 
Cancho GB, García FMS, Simal GJ (2000) El uso de los antibióticos en la alimentación animal: Perspectiva actual. Ciencia y Tecnologia Alimentaria 3: 39-47.

Castaldo A, Acero R, Perea J, Martos J, Valerio D, Pami J, García, A (2006) Tipología de los sistemas de producción de engorde bovino en la Pampa Argentina. Archivos de Zootecnia 55: 183-193.

Castro M, Rodríguez F (2005) Levaduras: probióticos y prebióticos que mejoran la producción animal. Revista Corpoica 6: 26-38.

Clemente I, Membrillo A, Azor PJ, Polvillo O, Juárez M, Santos E, Jiménez AM, Diéguez E, Molina A (2008) Caracterización de la diversidad genética intrarracial del cerdo lbérico. Información Técnica Económica Agraria 104: 314-322

Contino-Esquijerosa Y, Herrera-González R, Ojeda-García F, Iglesias-Gómez JM, Martín-Martín GJ (2017) Evaluación del comportamiento productivo en cerdos en crecimiento alimentados con una dieta no convencional. Pastos y Forrajes 40: 152-157

Díaz CA, Rodríguez MN, Vera VJ, Ramírez G, Casas GA, Mogollón JD (2011) Caracterización de los sistemas de producción porcina en las principales regiones porcicolas colombianas. Revista Colombiana de Ciencias Pecuarias 24: 131-144

FAO (2011) Molecular genetic characterization of animal genetic resources. Organización de la Naciones Unidas para la Agricultura y Alimentación. FAO Animal Production and Health Guidelines. Rome, Italy. 85p.

FAO (2012) Phenotypic characterization of animal genetic resources. Organización de la Naciones Unidas para la Agricultura y Alimentación. FAO Animal Production and Health Guidelines. Rome, Italy. 142p.

FAO (2014) Cerdos y la producción animal. Organización de la Naciones Unidas para la Agricultura y Alimentación. http://www.fao.org/ag/againfo/themes/es/pigs/production.html. Fecha de consulta: 10 de octubre de 2020.

FAO (2007) Plan de acción mundial sobre los recursos zoogenéticos y la declaración de Interlaken. Comisión de recursos genéticos para la alimentación y la agricultura. Organización de las Naciones Unidas para la Agricultura y la Alimentación. Roma, Italia. 40p.

Fuentes-Mascorro G, García HM, Altamirano ZA (2011) Centro de conservación y reproducción de cerdos criollos (CeCoRCeC). Revista Facultad de Agronomía LUZ 28: 586-599

García CH, Calle LM (1998) Consideraciones metodológicas para la tipificación de sistemas de producción bovina a partir de fuentes secundarias Corpoica. Ciencia y Tecnología Agropecuaria 2: 6-15.

Gelasakis AI, Valergakis GE, Arsenos G, Banos G (2012) Description and typology of intensive Chios dairy sheep farms in Greece. Journal of Dairy Science 95: 3070-3079.

Hernández-Baca M, Gámez-Rivas A, Zeledón-Ortega Y (2017) Caracterización morfológica del cerdo criollo (Sus scrofa domesticus) en el municipio de Nueva Guinea, RACCS. Revista Científica La Calera 17: 21-27.

Lemus C, Alonso MR, Alonso-Spilbury M, Ramírez NR (2003) Características morfológicas en cerdos nativos mexicanos. Archivos de Zootecnia 52: 105-108

Lemus C, LJ (2010) Estudios de sostenibilidad de cerdos mexicanos pelones y cuinos. La iniciativa nayarita. Revista Computadorizada de Producción Porcina 17: 89-98

Lemus-Flores C, Alonso-Morales R, Toledo-Alvarado H, Sansor-Nah R, Burgos-Paz W, Dzib-Cauich D (2020) Diversidad genética y estructura poblacional del cerdo negro lampiño de Yucatán usando chip SNP50. Abanico Veterinario 10: 1-12. DOI: 10.21929/abavet2020.10. 
Lesliea E, Geongb M, Abdurrahmanc M, Warda MP, Toribio JALML (2015) A description of smallholder pig production systems ineastern Indonesia. Preventive Veterinary Medicine 118: 319-327.

Lorenzo M, Jáuregui J, Vásquez Ch (2012) Caracterización del cerdo criollo de la Región Cho'rti' del Departamento de Chiquimula, Guatemala. Actas Iberoamericanas de Conservación Animal 2: 103-108.

Maroto-Molina F, Gómez-Cabrera A, Guerrero-Ginel JE, Garrido-Varo A, Adame-Siles JA, Pérez-Marín DC (2018) Caracterización y tipificación de explotaciones de dehesa asociadas a cooperativas: un caso de estudio en España. Revista Mexicana de Ciencias Pecuarias 9: 811-832.

Martínez VG, Román PSI, Vélez IA, Cabrera TE, Cantú CA, De la Cruz CL, Durán AM, Maldonado JJA, Martínez SFE, Ríos UA, Vega MVE, Ruiz LFJ (2016) Morfometría del cerdo de traspatio en áreas rurales de México. Revista Mexicana de Ciencias Pecuarias 7: 431-440.

Mireles S, Guerrero L, LJ (2015) Cerdos locales mexicanos: presencia y perspectivas del cerdo cuino. Revista Computadorizada de Producción Porcina 22: 115-125

Morales R, Rebatta M, Lucas J, Mateo J, Ramos D (2014) Caracterización de la crianza no tecnificada de cerdos en el parque porcino del distrito de Villa el Salvador, Lima-Perú. Salud y Tecnología Veterinaria 2: 39-48

Ogata N (2019) 1519, Hernán Cortés y el Cerdo en México. Diversidad Biológica y Cultural Trópico Americano. Centro de Investigaciones Tropicales (CITRO), Universidad Veracruzana. http://etnoecologia.uv.mx/ diversidad_biocultural/cerdo-pelon-mexicano/. Fecha de consulta: 10 de octubre de 2020.

Paixão G, Esteves A, Payan-Carreira R (2018) Characterization of a non-industrial pig production system: the case of Bísaro breed. Revista Brasileira de Zootecnia 47: e20170331. DOI: 10.1590/rbz4720170331.

Pérez F, Sierra AC, Canul MA, Ortiz JR, Bojórquez CJ, Rodríguez JC, Tamayo-Canul J (2015) Caracterización etnológica del cerdo pelón en el estado de Yucatán, México. Actas Iberoamericanas de Conservación Animal 6: 443-451.

Perezgrovas GR, Pérez AR, Galdámez FD (2007) Caracterización del sistema de cría de cerdos criollos en el contexto social de Aguacatenango, Chiapa. Quehacer Científico en Chiapas 3: 5-12.

Petrović M, Savić R, Parunović N, Dragan Radojković D, Radović C (2013) Reproductive traits of pigs of mangalitsa breed. Acta argiculturae Slovenica. Suppl. 4: 89-92.

Pujada AHN, Maguiña MRM, Luis ODB, Airahuacho BFE (2018) Caracterización morfológica del cerdo criollo Alto Andino. Infinitum 8: 23 -32.

Ramírez-Reyes JM, García-Robles E, Medellín-Cazares A, Osorto-Hernández W, Domínguez-Viveros J (2020) Caracterización genética y fenotípica de una población de cerdo pelón mexicano. Archivos de Zootecnia 69: 398-404.

Riedel S, Schiborra A, Hülsebusch C, Schlecht E (2014) The productivity of traditional smallholder pig production and posible improvement strategies in Xishuangbanna, South Western China. Livestock Science 160: 151162.

Salamanca-Carreño A, Jordana-Vidal J, Crosby-Granados RA, Arias-Landazábal JN, Parés-Casanova PM (2020) Sexual allometric monomorphism in araucan pig from Colombia: Preliminary results. Animals 10: 1763. DOI: 10.3390/ani10101763.

Savón LL (2014) Tropical roughage meals: effects on some physiological indicators in pigs. Revista Computadorizada de Producción Porcina 21: 99-108. 
Schembri N, Hernandez-Jovera M, Toribio ALMLJ, Holyoake PK (2015) On-farm characteristics and biosecurity protocols forsmall-scale swine producers in eastern Australia. Preventive Veterinary Medicine 118: 104-116.

Sierra-Vásquez AC, Ortiz-Ortiz JR, Bojórquez-Cat JC, Canul-Solís MA, Tamayo-Canul J R, Rodríguez-Pérez JC, Sanginés-García JR, Magaña-Magaña MA, Montes-Pérez RC, Segura-Correa JC (2016) Conservación y uso sustentable del cerdo pelón en Yucatán. Quehacer Científico en Chiapas. 11: 13-28.

Suárez-Mota ME, Villaseñor JL, López-Mata L (2017) Dominios climáticos de la Sierra Madre Oriental y su relación con la diversidad florística. Revista Mexicana de Biodiversidad 88: 224-233.

Vargas J, Velázquez FJ, Galíndez R, Pérez PE (2015) Estructura y relaciones genéticas del cerdo criollo de Ecuador. Revista Electrónica de Veterinaria 16: 1-12 